\title{
Numerical Treatment of a Two-Dimensional Vertically-Averaged Groundwater Flow Model Using Alternating Direction Explicit Methods
}

\author{
Supawan Yena ${ }^{1,2}$, Nopparat Pochai ${ }^{1,2, *}$ \\ ${ }^{1}$ Department of Mathematics, Faculty of Science, King Mongkut's Institute of Technology Ladkrabang, Thailand \\ ${ }^{2}$ Centre of Excellence in Mathematics, Commission on Higher Education (CHE), Thailand
}

Received February 14, 2020; Revised March 27, 2020; Accepted April 19, 2020

Copyright $\bigcirc 2020$ by authors, all rights reserved. Authors agree that this article remains permanently open access under the terms of the Creative Commons Attribution License 4.0 International License

\begin{abstract}
Leachate from a landfill can flow down and contaminate groundwater. Mathematical models are often used to describe groundwater flow, which can help designers to identify an appropriate location for a landfill area. We focused on simulation of hydraulic head of groundwater under landfill construction in a rural area. We used a partial differential equation model called two-dimensional vertically-averaged groundwater flow model to explain groundwater volume, in particular, to explain the hydraulic head of the groundwater which strongly affected the groundwater quality. We applied two alternating direction explicit methods to the groundwater flow model under general boundary conditions and rates of change of boundary conditions to obtain an approximate hydraulic head of groundwater. An alternating direction explicit method for all types of boundaries is used to find approximate solutions. The traditional alternative direction explicit method was modified to form our modified alternative direction explicit method. The results of all method are closed. The traditional alternative direction explicit method and the modified alternative direction explicit method led to more accurate solutions than forward time centered space in heterogeneous aquifer, two methods ensured that a stable solution of all interior points can be obtained by using a fine grid spacing.
\end{abstract}

Keywords Groundwater Model, Finite Difference Method, Alternating Direction Explicit Method

\section{Introduction}

Pollutants in groundwater can alter an aquatic ecosystem. A major groundwater deterioration problem is contamination of chemicals and heavy metals. Industrial development often causes many pollution problems. Leachate of toxins and solid waste from landfills and fertilizers from agricultural activities can introduce pollutants into groundwater. Agricultural activities may cause the nitrate level in groundwater to exceed standards. Contaminated ground water produces detrimental effects on health. Groundwater may become contaminated by leaching of nitrate from fertilizer on agricultural fields and waste dumps in rural and urban areas. In general, nitrate decreases the ability of blood cells to carry oxygen, resulting in oxygen deficiency in some body parts that can be linked to blue baby syndrome [1]. Groundwater source contamination renders plants, humans, and animals unsustainable. Groundwater deterioration may also come from seawater intrusion in coastal areas. Salinity contamination in groundwater in a rice field near a marine shrimp aquaculture farm can lead to poor crop production [2]-[3]. Even though bacteria, fungi, and other such biological pollutants are naturally filtered out or diluted as water percolates down through soil and rocks, in recent years, this groundwater natural defense system has been vastly overextended. The sheer volume of biological pollutants sent underground has escalated, especially in the last century, when global population increased rapidly and demands for food and water grew enormously. Its effects are very serious especially for people who rely on groundwater during periods of drought. At the same time, industries are quickly advancing, further contaminating the environment. Rivers and streams are also damaged by human consumption. Whenever water flows over the ground surface, the water will be damaged and consequently affects the quality of the groundwater, since groundwater is from surface water accumulated underground, which is usually filtered clean by the ground. Groundwater exists mainly in aquifers, bodies of water-saturated rock, through which water can move or in 
which it can stay. Since underground water is not clearly visible, we cannot determine its movement direction, if we do not dig and drill to it. Darcy was interested in describing groundwater flow and created a mathematical model Darcy's law [5], which can describe underground water flow. We modeled groundwater flow based on Darcy's law. Ground water systems can be modeled by using partial differential equations, usually numerically solved by finite difference and finite element methods. There have been several studies related work on groundwater modeling simulation with finite difference methods [6] and spreadsheets [7]-[8] or MODFLOW [9]. Spreadsheets can be designed to mimic some packages in MODFLOW. An explicit finite difference method is easier to solve than an implicit one. An explicit finite difference scheme for solving nonlinear time-dependent equations can be applied to linear equations. This scheme has been proven to be second order accurate in time and unconditionally stable for arbitrary time step size [10]. An unconditionally stable alternating direction explicit scheme (ADE) for solving one-dimensional unsteady convection-diffusion equation was developed Xie et al [11].Apart from being explicit and unconditionally stable, the method is straightforward to implement [12].

Here, we introduce a two-dimensional verticallyaveraged groundwater flow model. It can determine the hydraulic head of groundwater in the considered area.We have compared our numerical solution,forward time centered space and alternating direction explicit method for all types of boundaries is used to find approximate solutions in homogeneous and heterogeneous aquifer.

\section{Groundwater Flow Model}

The governing equations of groundwater flow based on Darcy's law are partial differential equations, described in section 2.1.

\subsection{A Two-Dimensional Vertically Averaged Groundwater Flow Model}

Groundwater flow can be described by a transient hydraulic head flow model. The aquifer is assumed to be a porous medium and assumed that water is uniformly transferred through the soil of a homogeneous aquifer. The groundwater flow model is used to represent the behavior of a hydraulic head. The transmission property is hydraulic conductivity through the considered space, including the aquifer and its space. Using Darcy's law, the hydraulic head in the considered area is assumed to be vertically averaged. The domain is defined to be $\Omega=\{(\mathrm{x}, \mathrm{y}) 0 \leq \mathrm{x} \leq \mathrm{I}, 0 \leq \mathrm{y} \leq \mathbf{J}\}$. see in Fig. 1. The governing equation of Darcy's flow is [6].
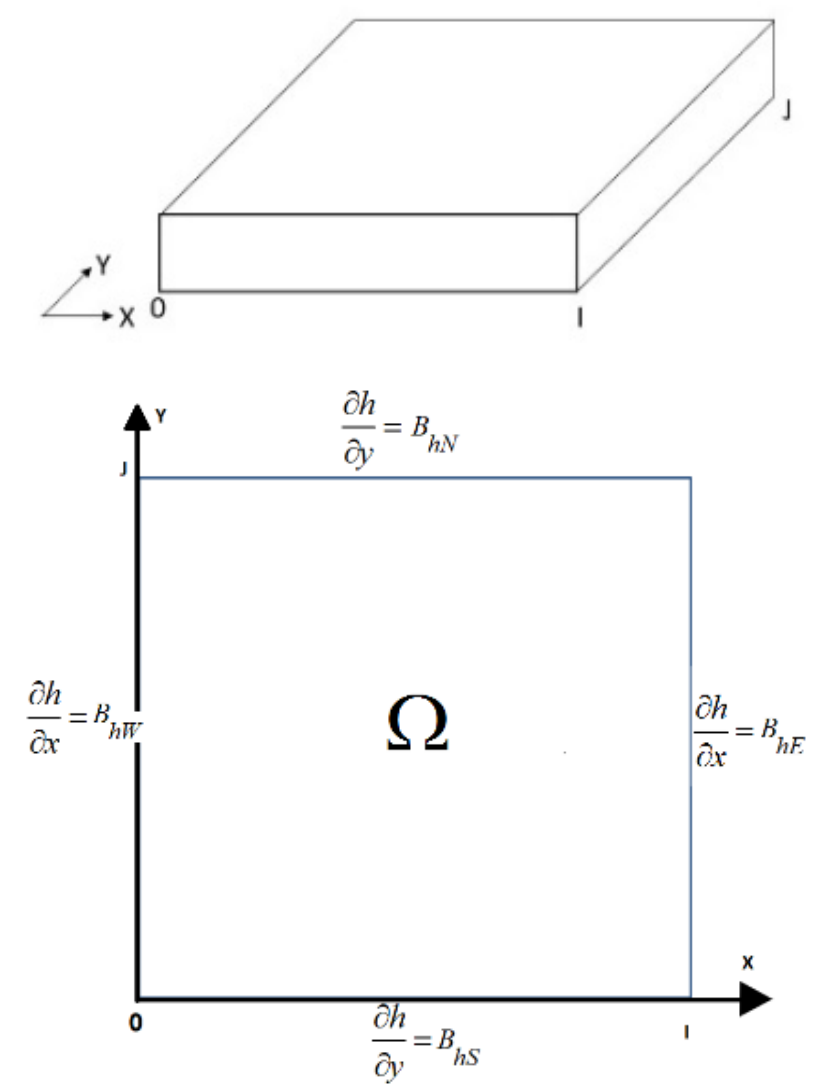

Figure 1. Underground topography of the considered area

$$
\frac{\partial}{\partial t}\left(K_{x} \frac{\partial h}{\partial t}\right)+\frac{\partial}{\partial t}\left(K_{y} \frac{\partial h}{\partial t}\right) \pm W=S \frac{\partial h}{\partial t}
$$

for all $(x, y, t) \in \Omega \times[0, T], h(x, y, t)(\mathrm{m})$ is the hydraulic head, $K_{x}(\mathrm{~m} /$ day $)$ and $K_{y}(\mathrm{~m} /$ day $)$ are the hydraulic conductivity values in the principal directions: $x$ - and $y$ direction, $W\left(\right.$ day $\left.^{-1}\right)$ is the source or sink function, $S\left(\mathrm{~m}^{-1}\right)$ is the specific storagec oefficient. In this and all following equations, we use SI units consistently.

If we consider a homogeneous aquifer system, the hydraulic conductivity value will be constant, $K_{x}=K_{y}$. Then (1) becomes

$$
K\left(\frac{\partial^{2} h}{\partial x^{2}}+\frac{\partial^{2} h}{\partial y^{2}}\right) \pm W=S \frac{\partial h}{\partial t}
$$

\subsubsection{Initial Conditions}

The initial condition of (2) is defined by the static potential hydraulic head,

$$
\mathrm{h}(\mathrm{x}, \mathrm{y}, 0)=\mathrm{h}_{0} \text { for all }(\mathrm{x}, \mathrm{y}) \in \Omega,
$$

where $h_{0}$ is a given average potential hydraulic head around the considered area.

\subsubsection{Boundary Conditions}

[1] The rates of change of the hydraulic head along the domain boundaries are given by 


$$
\begin{array}{r}
\frac{\partial h}{\partial y}(x, J, t)=B_{h N} \text { for all } 0 \leq x \leq I, t>0 \text {.(3) } \\
\frac{\partial h}{\partial y}(x, 0, t)=B_{h S} \text { for all } 0 \leq x \leq I, t>0 . \\
\frac{\partial \mathrm{h}}{\partial \mathrm{y}}(\mathrm{x}, 0, \mathrm{t})=\mathrm{B}_{\mathrm{hS}}, \text { forall } 0 \leq \mathrm{x} \leq \mathrm{I}, \mathrm{t}>0, \frac{\partial \mathrm{h}}{\partial \mathrm{y}}(\mathrm{x}, 0, \mathrm{t})= \\
\mathrm{B}_{\mathrm{hS}} \text {, forall } 0 \leq \mathrm{x} \leq \mathrm{I}, \mathrm{t}>0,(4) \\
\frac{\partial h}{\partial x}(0, y, t)=B_{h W} \text { for all } 0 \leq y \leq J, t>0 .(5) \\
\frac{\partial h}{\partial x}(I, y, t)=B_{h E} \text { for all } 0 \leq y \leq J, t>0 .(6)
\end{array}
$$

where $B_{h N}, B_{h S}, B_{h E}$ and $B_{h W}$ are the rates of change of hydraulic head on the north, south, west and east domain boundaries - see Fig. 1.

\section{Explicit finite Difference Techniques}

Finite difference methods are numerical methods for solving differential equations by approximating them with difference equations, in which finite differences approximate the derivatives. A finite difference method is said to be explicit when it can be computed forward in time using quantities from previous time steps. In the explicit finite difference method of (2), the hydraulic head at time $n+1$ depends explicitly on the head at time $n$.

We now discretize the domain in Fig. 1 by dividing the interval $[0, I]$ into $M$ subintervals, so that $M \Delta x=I$ similarly divide $[0, J]$ and $[0, T]$ into $N$ and $P$ subintervals, $N \Delta y=J$ and $P \Delta t=T$. We can then approximate $h\left(x_{i}, y_{j}, t_{n}\right)$ by $h_{i, j}^{n}$, value of the difference approximation of $h(x, y, t)$ at point $x_{i}$ $=i \Delta x, y_{j}=j \Delta y$ and $t_{n}=n \Delta t$, when $0 \leq i \leq M, 0 \leq j \leq N$ and $0 \leq$ $n \leq P$, where $M, N$ and $P$ are positive integers.

\subsection{Forward Time Centered Space (FTCS)}

We introduced the forward time centered space technique to a two-dimensional vertically averaged groundwater flow model (2), we obtained that

$$
\begin{gathered}
\frac{\partial h}{\partial t} \approx \frac{h_{i, j}^{n+1}-h_{i, j}^{n}}{\Delta t} . \\
\frac{\partial^{2} h}{\partial x^{2}} \approx \frac{h_{i+1, j}^{n}-2 h_{i, j}^{n}+h_{i-1, j}^{n}}{(\Delta x)^{2}} . \\
\frac{\partial^{2} h}{\partial y^{2}} \approx \frac{h_{i, j+1}^{n}-2 h_{i, j}^{n}+h_{i, j-1}^{n}}{(\Delta y)^{2}} .
\end{gathered}
$$

$$
\begin{gathered}
K\left(\frac{h_{i+1, j}^{n}-2 h_{i, j}^{n}+h_{i-1, j}^{n}}{(\Delta x)^{2}}+\frac{h_{i, j+1}^{n}-2 h_{i, j}^{n}+h_{i, j-1}^{n}}{(\Delta y)^{2}}\right) \\
\pm W=S\left(\frac{h_{i, j}^{n+1}-h_{i, j}^{n}}{\Delta t}\right),
\end{gathered}
$$

for all when $0 \leq \mathrm{i} \leq \mathrm{M}, 0 \leq \mathrm{j} \leq \mathrm{N}$ and $0 \leq \mathrm{n} \leq \mathrm{P}$.

Rearranging (10), we have

$$
\begin{aligned}
h_{i, j}^{n+1}= & \alpha\left(h_{i+1, j}^{n}-2 h_{i, j}^{n}+h_{i-1, j}^{n}\right) \\
& +\beta\left(h_{i, j+1}^{n}-2 h_{i, j}^{n}+h_{i, j-1}^{n}\right)+\gamma+h_{i, j}^{n},
\end{aligned}
$$

where $\alpha=\frac{K \Delta t}{S(\Delta x)^{2}}, \beta=\frac{K \Delta t}{S(\Delta y)^{2}} \alpha=\frac{K \Delta t}{S(\Delta x)^{2}}, \beta=\frac{K \Delta t}{S(\Delta y)^{2}}$ and $\gamma=\frac{W \Delta t}{s}$.

\subsection{Alternating Direction Explicit Methods (ADEM) for a Two-Dimensional Vertically Averaged Groundwater Flow Model}

The alternating direction explicit scheme consists of three steps. We use the alternating direction explicit method to approximate the solution of (2) to obtain the finite difference discretization:

\subsubsection{Traditional Alternating Direction Explicit Method (ADEM)}

We introduced the traditional alternating direction explicit method to a two-dimensional vertically averagedgroundwater flow model (2).

3.2.1.1. Initialization

$$
\begin{aligned}
h_{i, j}^{n} & =h 1_{i, j}^{n} . \\
h_{i, j}^{n} & =h 2_{i, j}^{n} .
\end{aligned}
$$

\subsubsection{First Stage}

$$
\begin{gathered}
\frac{\partial h}{\partial t} \approx \frac{h 1_{i, j}^{n+1}-h 1_{i, j}^{n}}{\Delta t} \\
\frac{\partial^{2} h}{\partial x^{2}} \approx \frac{h 1_{i-1, j}^{n+1}-h 1_{i, j}^{n+1}-h 1_{i, j}^{n}+h 1_{i+1, j}^{n}}{(\Delta x)^{2}} \\
\frac{\partial^{2} h}{\partial y^{2}} \approx \frac{h 1_{i, j-1}^{n+1}-h 1_{i, j}^{n+1}-h 1_{i, j}^{n}+h 1_{i, j+1}^{n}}{(\Delta y)^{2}}
\end{gathered}
$$

Substituting (14)-(16) into (2), we obtain

Substituting (7)-(9) into (2), we obtain 


$$
\begin{aligned}
& K x_{i, j}\left(\frac{h 1_{i-1, j}^{n+1}-h 1_{i, j}^{n+1}-h 1_{i, j}^{n}+h 1_{i+1, j}^{n}}{(\Delta x)^{2}}\right) \\
& +K y_{i, j}\left(\frac{h 1_{i, j-1}^{n+1}-h 1_{i, j}^{n+1}-h 1_{i, j}^{n}+h 1_{i, j+1}^{n}}{(\Delta y)^{2}}\right) \\
& \pm W=S\left(\frac{h 1_{i, j}^{n+1}-h 1_{i, j}^{n}}{\Delta t}\right)
\end{aligned}
$$

Then the explicit finite difference equation becomes

$$
\begin{aligned}
h 1_{i, j}^{n+1}= & \frac{\alpha h 1_{i-1, j}^{n+1}+\beta h 1_{i, j-1}^{n+1}-(\alpha+\beta-1) h 1_{i, j}^{n}}{1+\alpha+\beta} \\
& +\frac{\alpha h 1_{i+1, j}^{n}+\beta h 1_{i, j+1}^{n}}{1+\alpha+\beta}+\gamma
\end{aligned}
$$

3.2.1.3. Second Stage

$$
\begin{gathered}
\frac{\partial h}{\partial t} \approx \frac{h 2_{i, j}^{n+1}-h 2_{i, j}^{n}}{\Delta t} . \\
\frac{\partial^{2} h}{\partial x^{2}} \approx \frac{h 2_{i+1, j}^{n+1}-h 2_{i, j}^{n+1}-h 2_{i, j}^{n}+h 2_{i-1, j}^{n}}{(\Delta x)^{2}} . \\
\frac{\partial^{2} h}{\partial y^{2}} \approx \frac{h 2_{i, j+1}^{n+1}-h 2_{i, j}^{n+1}-h 2_{i, j}^{n}+h 2_{i, j-1}^{n} .}{(\Delta y)^{2}} .
\end{gathered}
$$

Substituting (18)-(20) into (2), we obtain

$$
\begin{aligned}
& K x_{i, j}\left(\frac{h 2_{i+1, j}^{n+1}-h 2_{i, j}^{n+1}-h 2_{i, j}^{n}+h 2_{i-1, j}^{n}}{(\Delta x)^{2}}\right) \\
& +K y_{i, j}\left(\frac{h 2_{i, j+1}^{n+1}-h 2_{i, j}^{n+1}-h 2_{i, j}^{n}+h 2_{i, j-1}^{n}}{(\Delta y)^{2}}\right) \\
& \pm W=S\left(\frac{h 2_{i, j}^{n+1}-h 2_{i, j}^{n}}{\Delta t}\right)
\end{aligned}
$$

Then the explicit finite difference equation becomes

$$
\begin{aligned}
h 2_{i, j}^{n+1}= & \frac{\alpha h 2_{i+1, j}^{n+1}+\beta h 2_{i, j+1}^{n+1}-(\alpha+\beta-1) h 2_{i, j}^{n}}{1+\alpha+\beta} \\
& +\frac{\alpha h 2_{i-1, j}^{n}+\beta h 2_{i, j-1}^{n}}{1+\alpha+\beta}+\gamma
\end{aligned}
$$

\subsubsection{Last Stage}

$$
h_{i, j}^{n+1}=\frac{h 1_{i, j}^{n+1}+h 2_{i, j}^{n+1}}{2} .
$$

where $\alpha=\frac{K x_{i, j} \Delta t}{S(\Delta x)^{2}}, \beta=\frac{K y_{i, j} \Delta t}{S(\Delta y)^{2}}$ and $\gamma=\frac{W \Delta t}{S}$.

3.2.2. Modified Alternating Direction Explicit Method
$\left(\mathrm{ADEM}_{\mathrm{M}}\right)$

We introduce the modified alternating direction explicit method to a two-dimensional vertically averaged groundwater flow model (2)

\subsubsection{Initialization}

$$
\begin{aligned}
& h_{i, j}^{n}=h 1_{i, j}^{n} . \\
& h_{i, j}^{n}=h 2_{i, j}^{n} .
\end{aligned}
$$

3.2.2.2. First Stage

$$
\begin{gathered}
\frac{\partial h}{\partial t} \approx \frac{h 1_{i, j}^{n+1}-h 1_{i, j}^{n}}{\Delta t} . \\
\frac{\partial^{2} h}{\partial x^{2}} \approx \frac{h 1_{i-1, j}^{n+1}-h 1_{i, j}^{n+1}-h 1_{i, j}^{n}+h 1_{i+1, j}^{n}}{(\Delta x)^{2}} . \\
\frac{\partial^{2} h}{\partial y^{2}} \approx \frac{h 1_{i, j-1}^{n+1}-h 1_{i, j}^{n+1}-h 1_{i, j}^{n}+h 1_{i, j+1}^{n}}{(\Delta y)^{2}} .
\end{gathered}
$$

Substituting (26) - (28) into (2), we obtain

$$
\begin{aligned}
& K x_{i+\frac{1}{2}, j}\left(\frac{h 1_{i+1, j}^{n}-h 1_{i, j}^{n}}{(\Delta x)^{2}}\right)+K x_{i-\frac{1}{2}, j}\left(\frac{h 1_{i-1, j}^{n+1}-h 1_{i, j}^{n+1}}{(\Delta x)^{2}}\right) \\
& +K y_{i, j+\frac{1}{2}}\left(\frac{h 1_{i, j+1}^{n}-h 1_{i, j}^{n}}{(\Delta y)^{2}}\right)+K y_{i, j-\frac{1}{2}}\left(\frac{h 1_{i, j-1}^{n+1}-h 1_{i, j}^{n+1}}{(\Delta y)^{2}}\right) \\
& \pm W=S\left(\frac{h 1_{i, j}^{n+1}-h 1_{i, j}^{n}}{\Delta t}\right)
\end{aligned}
$$

Then the explicit finite difference equation becomes

$$
\begin{aligned}
h 1_{i, j}^{n+1}= & \frac{\alpha_{2} h 1_{i-1, j}^{n+1}+\beta_{2} h 1_{i, j-1}^{n+1}-\left(\alpha_{1}+\beta_{1}-1\right) h 1_{i, j}^{n}}{1+\alpha_{2}+\beta_{2}} \\
& +\frac{\alpha_{1} h 1_{i+1, j}^{n}+\beta_{1} h 1_{i, j+1}^{n}}{1+\alpha_{2}+\beta_{2}}+\gamma
\end{aligned}
$$

where $\quad \alpha_{1}=\frac{K x_{i+\frac{1}{2}, j} \Delta t}{S(\Delta x)^{2}}, \quad \alpha_{2}=\frac{K x_{i-\frac{1}{2}, j} \Delta t}{S(\Delta x)^{2}}, \quad \beta_{1}=\frac{K y_{i, j+\frac{1}{2}} \Delta t}{S(\Delta y)^{2}}$, $\beta_{2}=\frac{K y_{i, j-\frac{1}{2}} \Delta t}{S(\Delta y)^{2}}$ and $\gamma=\frac{W \Delta t}{S}$.

3.2.2.3. Second Stage

$$
\begin{gathered}
\frac{\partial h}{\partial t} \approx \frac{h 2_{i, j}^{n+1}-h 2_{i, j}^{n}}{\Delta t} . \\
\frac{\partial^{2} h}{\partial x^{2}} \approx \frac{h 2_{i-1, j}^{n+1}-h 2_{i, j}^{n+1}-h 2_{i, j}^{n}+h 2_{i+1, j}^{n}}{(\Delta x)^{2}} \\
\frac{\partial^{2} h}{\partial y^{2}} \approx \frac{h 2_{i, j-1}^{n+1}-h 2_{i, j}^{n+1}-h 2_{i, j}^{n}+h 2_{i, j+1}^{n}}{(\Delta y)^{2}}
\end{gathered}
$$


Substituting (29) - (31) into (2), we obtain

$$
\begin{aligned}
& K x_{i-\frac{1}{2}, j}\left(\frac{h 2_{i-1, j}^{n}-h 2_{i, j}^{n}}{(\Delta x)^{2}}\right)+K x_{i+\frac{1}{2}, j}\left(\frac{h 2_{i+1, j}^{n+1}-h 2_{i, j}^{n+1}}{(\Delta x)^{2}}\right) \\
& +K y_{i, j-\frac{1}{2}}\left(\frac{h 2_{i, j-1}^{n}-h 2_{i, j}^{n}}{(\Delta y)^{2}}\right)+K y_{i, j+\frac{1}{2}}\left(\frac{h 2_{i, j+1}^{n+1}-h 2_{i, j}^{n+1}}{(\Delta y)^{2}}\right) \\
& \pm W=S\left(\frac{h 2_{i, j}^{n+1}-h 2_{i, j}^{n}}{\Delta t}\right)
\end{aligned}
$$

Then the explicit finite difference equation becomes

$$
\begin{aligned}
h 2_{i, j}^{n+1}= & \frac{\alpha_{2} h 2_{i+1, j}^{n+1}+\beta_{2} h 2_{i, j+1}^{n+1}-\left(\alpha_{1}+\beta_{1}-1\right) h 2_{i, j}^{n}}{1+\alpha_{2}+\beta_{2}} \\
& +\frac{\alpha_{1} h 2_{i-1, j}^{n}+\beta_{1} h 2_{i, j-1}^{n}}{1+\alpha_{2}+\beta_{2}}+\gamma
\end{aligned}
$$

3.2.2.4. Last Stage

$$
h_{i, j}^{n+1}=\frac{h 1_{i, j}^{n+1}+h 2_{i, j}^{n+1}}{2} .
$$

\subsubsection{Total Mass Error (TME)}

The precision of our proposed numerical techniques is tested using the total mass error [7]. The total mass error, as defined below, is used to quantify the effect of heterogeneous aquifer complexity.

$$
T M E=\frac{\mid \sum \text { Mass before Calculate }-\sum \text { Mass after Calculate } \mid}{\sum \text { Mass before Calculate } \times T} \times 100
$$

\section{Numerical Simulations of a Two-Dimensional Vertically Averaged Groundwater Flow Model Model under Several Boundary Conditions with Forward Time}

\section{centered Space, Traditional and Modified Alternating Direction Explicit Method}

We introduce a two-dimensional vertically- averaged groundwater flow model.It can determine the hydraulic head and showed the flow velocity of groundwater, entered to all directions underground in the considered area.

\subsection{Simulation 1: Comparing Numerical Solutions}

We considered a $10 \times 10 \mathrm{~km}$ area of groundwater - see Fig. 2.

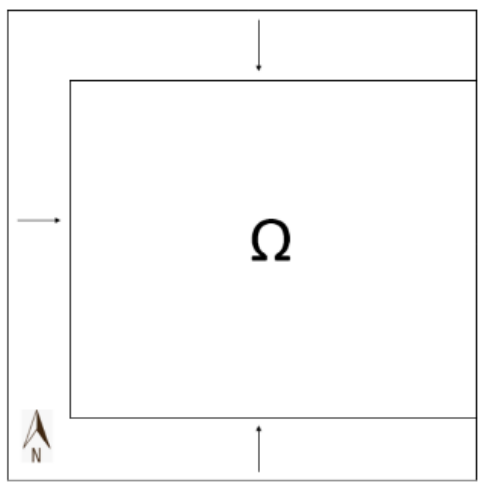

Figure 2. Area of simulation 1

Assumed that the rate of change of hydraulic head along the west boundary is $-0.1(\mathrm{~m})$. Similarly, the rate of change along the north, south and east boundaries are $-0.1(\mathrm{~m}), 0.1$ (m) and $0.0(\mathrm{~m})$ respectively. The potential hydraulic head is $0(\mathrm{~m})$. The hydraulic conductivity is $1.5(\mathrm{~m} / \mathrm{day})$. The storage capacity is $10\left(\mathrm{~m}^{-1}\right)$. We discretize the domain by dividing the interval, $\Delta x=1, \Delta y=1$ and $\Delta t=1$ for all $0 \leq x \leq 10$, $0 \leq y \leq 10$ and $0 \leq z \leq 3600$. The parameters used are set out in Table 1.

We computed the hydraulic head over 10 years, $h(x, y, 3600)$. Solutions for FTCS, ADEM and $\mathrm{ADEM}_{\mathrm{M}}$ are shown in Fig. 3 - Fig. 5.and graph showing the trend for the three methods in Fig 6.

Table 1. Parameters for simulation 1: Groundwater hydraulic head

\begin{tabular}{|c|c|c|c|c|c|c|c|c|c|c|}
\hline $\mathrm{K}$ & $\mathrm{S}$ & $\mathrm{W}$ & $\mathrm{h}(\mathrm{x}, \mathrm{y}, 0)$ & $\mathrm{B}_{\mathrm{hW}}$ & $\mathrm{B}_{\mathrm{hE}}$ & $\mathrm{B}_{\mathrm{hs}}$ & $\mathrm{B}_{\mathrm{hN}}$ & $\Delta \mathrm{x}$ & $\Delta \mathrm{y}$ & $\Delta \mathrm{t}$ \\
\hline 1.5 & 10 & 0.0 & 0.0 & -0.1 & 0.0 & 01 & -0.1 & 1.0 & 1.0 & 1.0 \\
\hline
\end{tabular}



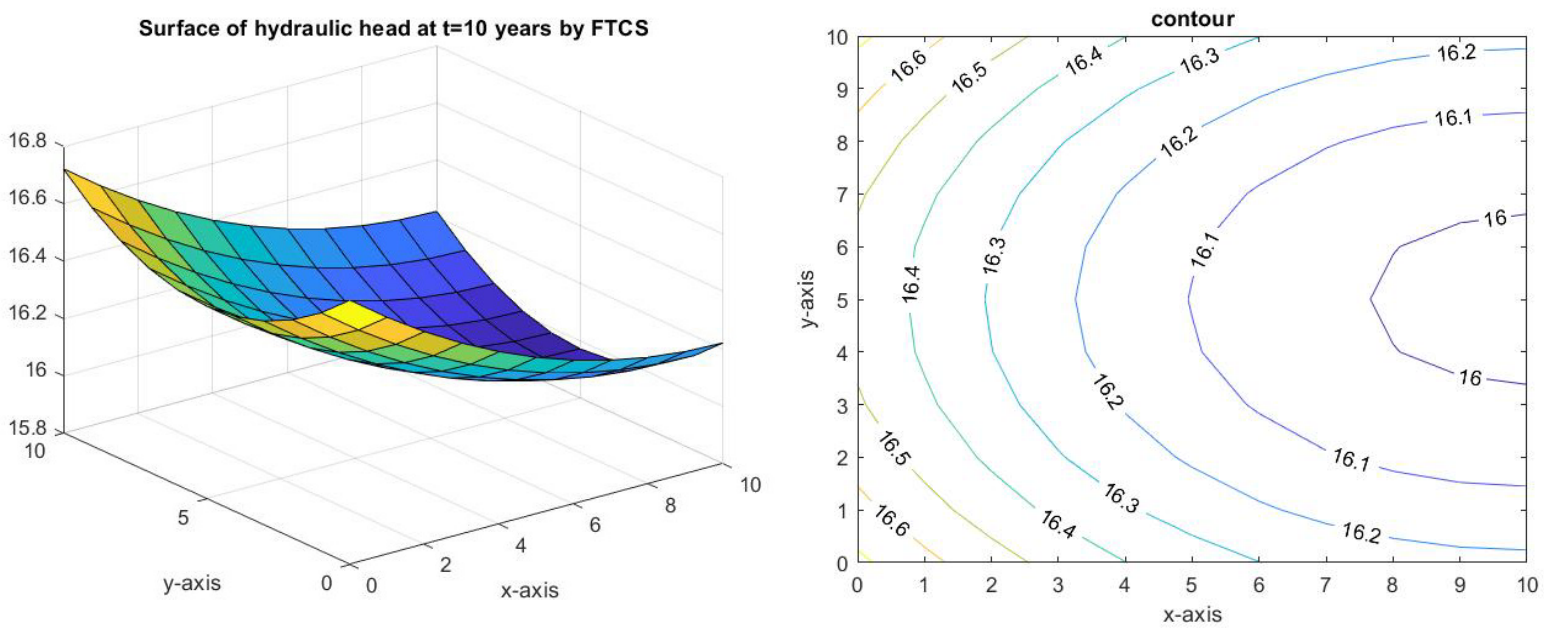

Figure 3. FTCS solution in simulation 1
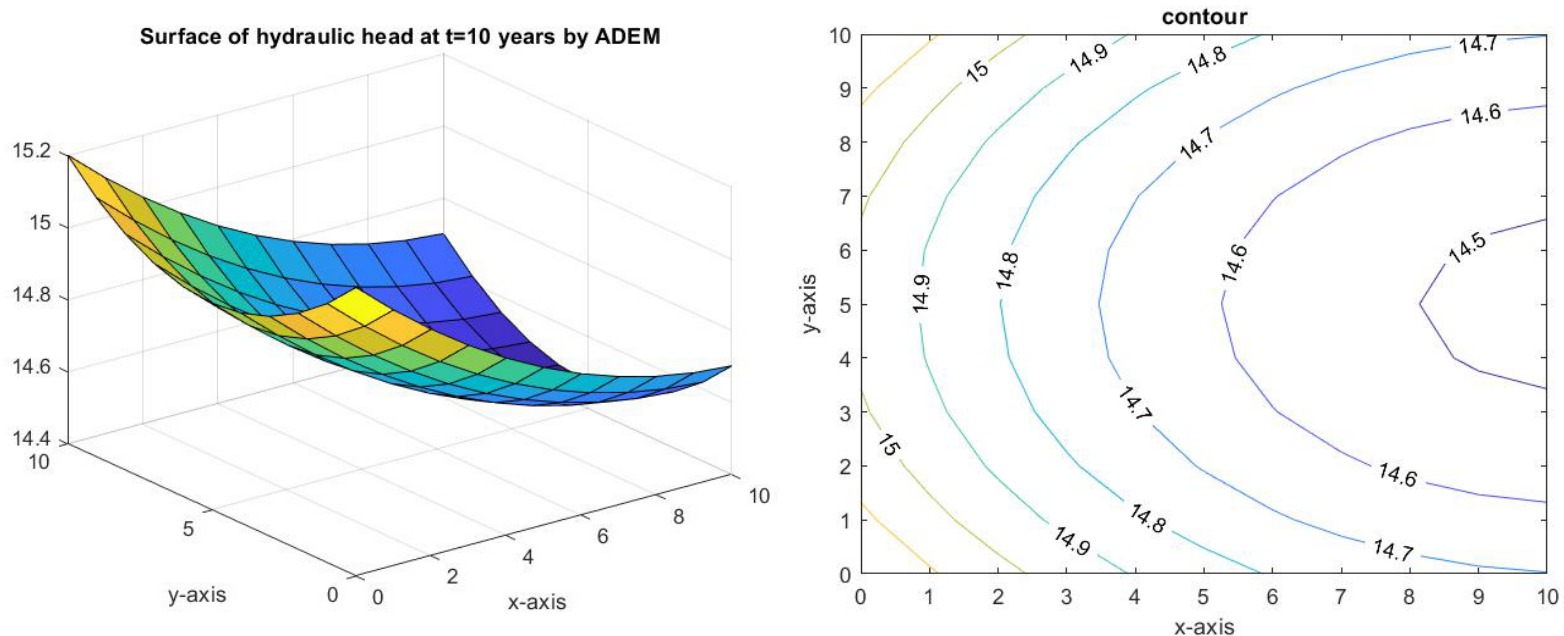

Figure 4. ADEM solution in simulation 1
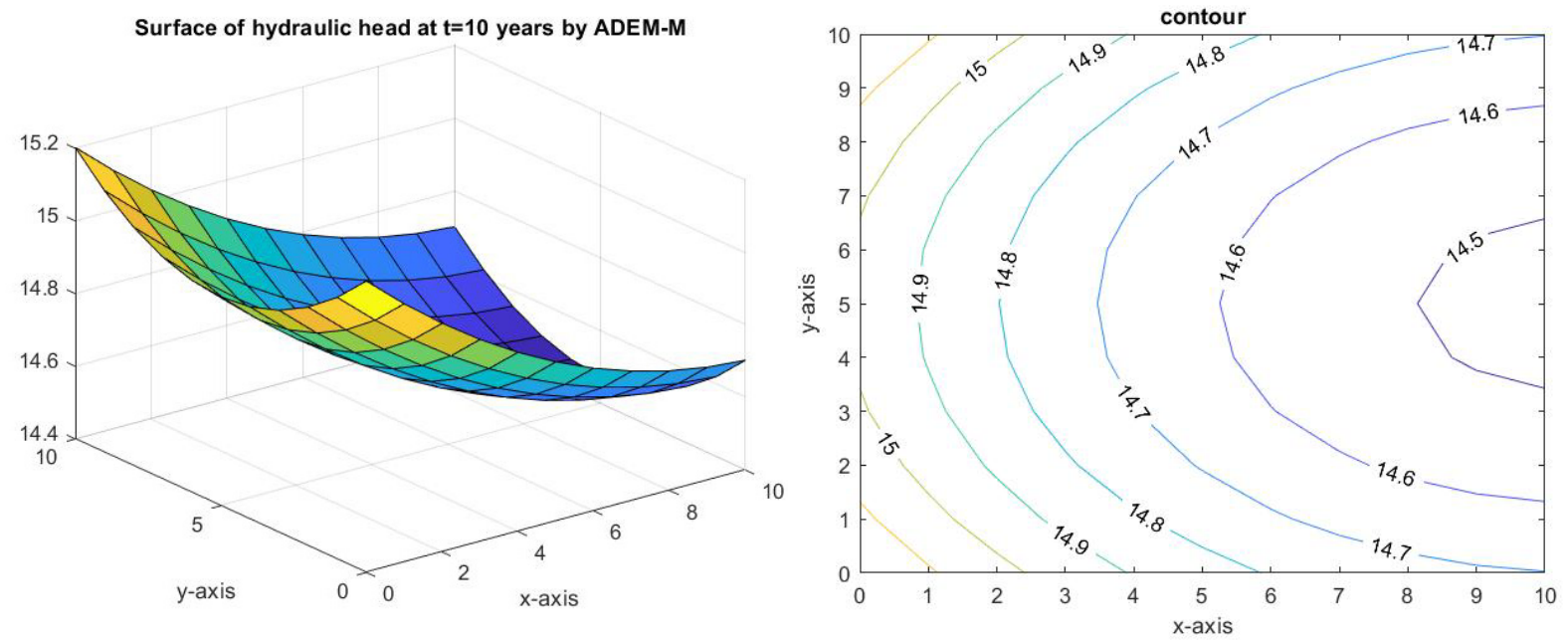

Figure 5. $\mathrm{ADEM}_{\mathrm{M}}$ solution in simulation1 


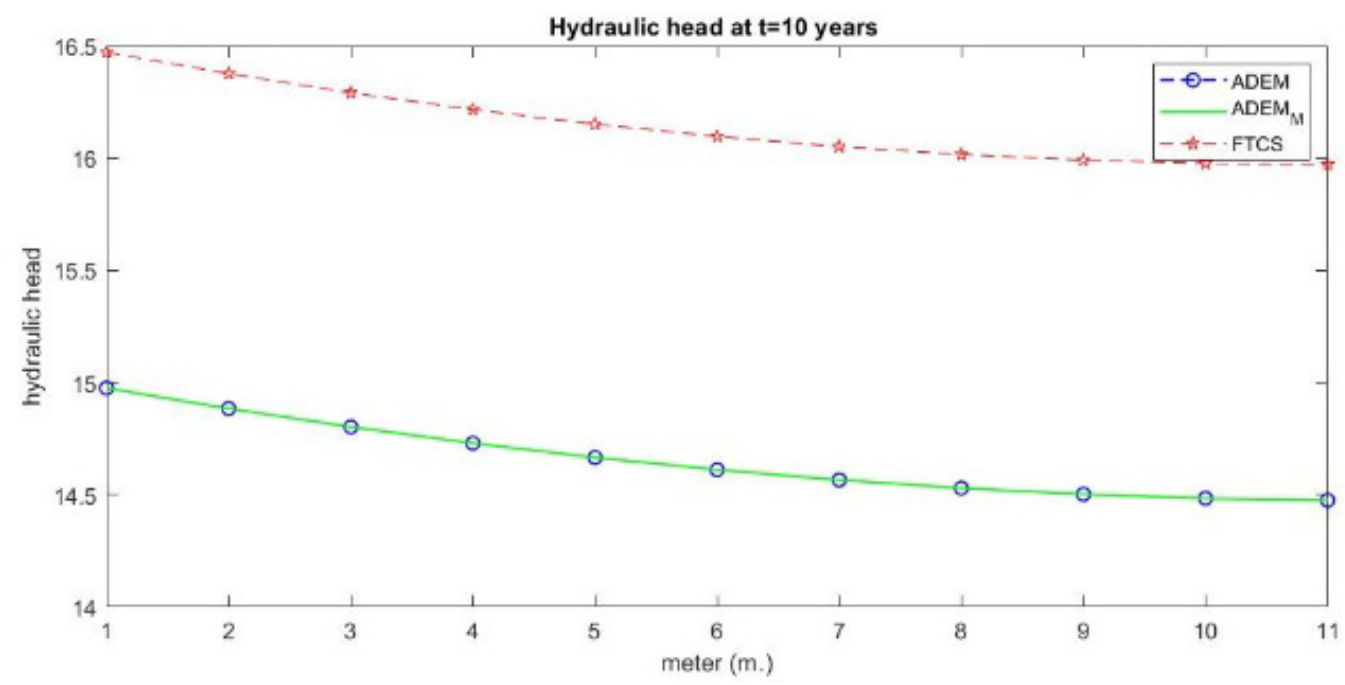

Figure 6. Hydraulic head vs meters for the three solutions

\subsection{Simulation 2: Convergence of Approximate Solutions}

Again, we considered a $10 \times 10(\mathrm{~km})$ area - see Fig. 1 .

Assumed that the rate of change of hydraulic head along the west boundary is $-0.1(\mathrm{~m})$. Similarly, the rate of change along the north, south and east boundaries are $-0.1(\mathrm{~m}),(0.1) \mathrm{m}$ and $0.0(\mathrm{~m})$ respectively. The potential hydraulic head is 0 (m). The hydraulic conductivity is $1.5\left(\mathrm{~m} /\right.$ day). The storage capacity is $10 \mathrm{~m}^{-1}$. The parameters used are set out in Table 2 .

Table 2. Parameters for simulation 2: Groundwater hydraulic head

\begin{tabular}{|c|c|c|c|c|c|c|c|c|c|c|}
\hline $\mathrm{K}$ & $\mathrm{S}$ & $\mathrm{W}$ & $\mathrm{h}(\mathrm{x}, \mathrm{y}, 0)$ & $\mathrm{B}_{\mathrm{hW}}$ & $\mathrm{B}_{\mathrm{hE}}$ & $\mathrm{B}_{\mathrm{hs}}$ & $\mathrm{B}_{\mathrm{hN}}$ & $\Delta \mathrm{x}$ & $\Delta \mathrm{y}$ & $\Delta \mathrm{t}$ \\
\hline 1.5 & 10 & 0.0 & 0.0 & -0.1 & 0.0 & 01 & -0.1 & 0.2 & 0.2 & 1.0 \\
\hline
\end{tabular}

We computed the hydraulic head over 10 years, $h(x, y, 3600)$ by changing the changing the grid spacing, $\Delta x=0.2$, $\Delta y=0.2$ and $\Delta t=1$ for all $0 \leq x \leq 10,0 \leq y \leq 10$ and $0 \leq z \leq 3600$. FTCS could not produce a stable solution, but solution for ADEM and $\mathrm{ADEM}_{\mathrm{M}}$ are illustrated in Fig.7 - Fig. 8.
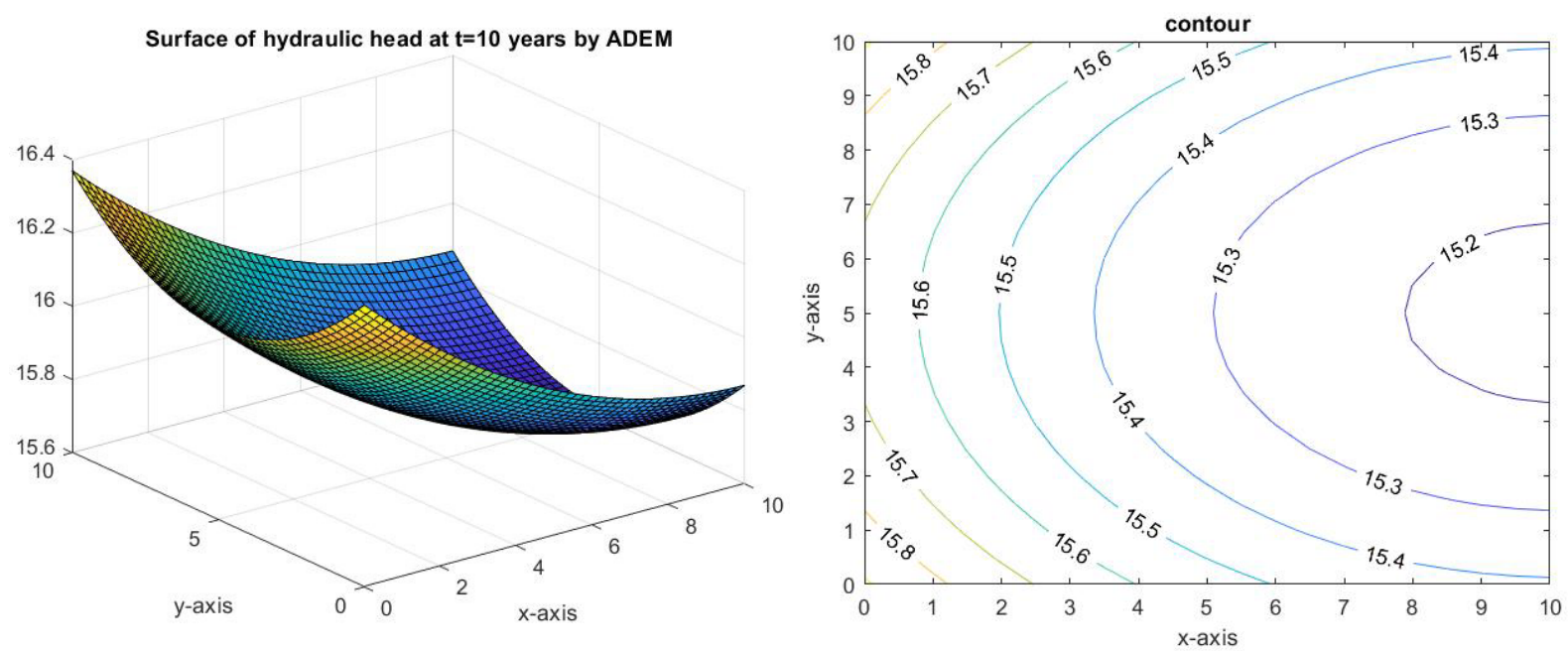

Figure 7. ADEM solution in simulation2 

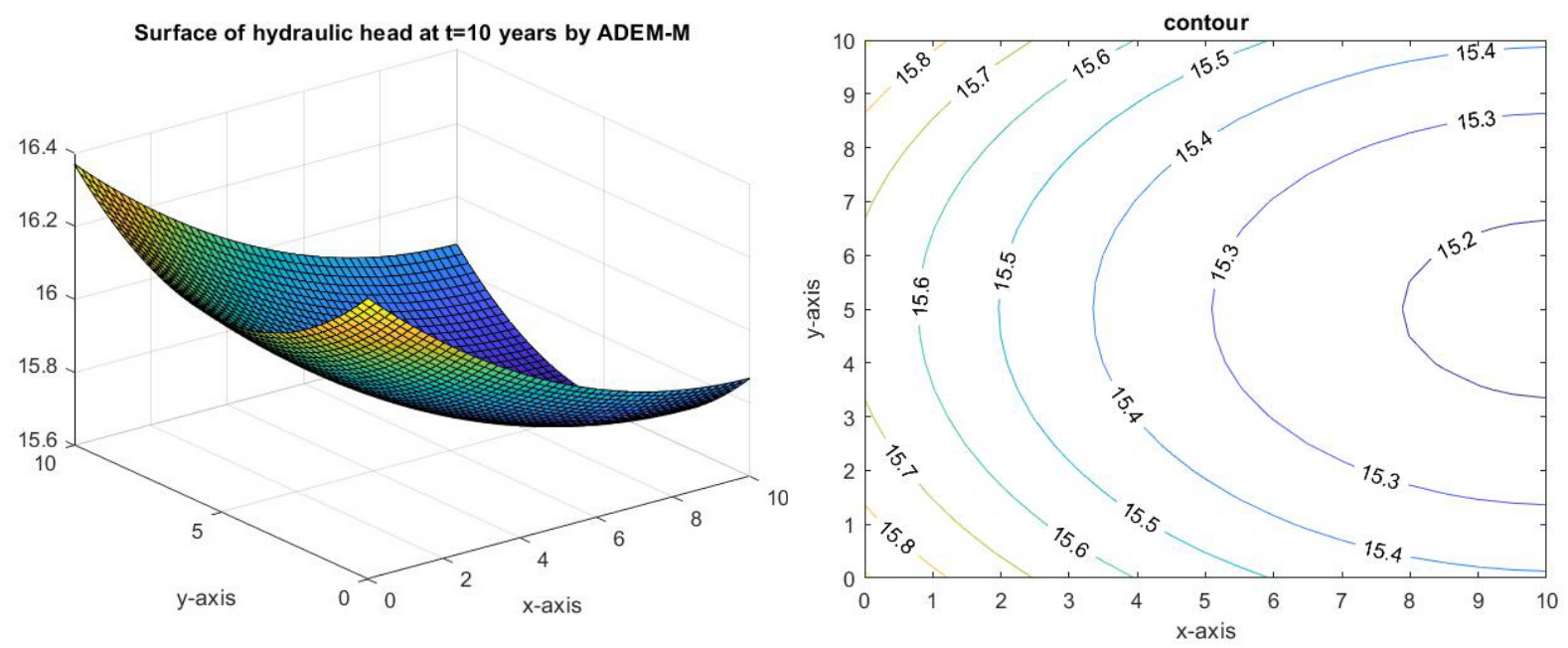

Figure 8. $\mathrm{ADEM}_{\mathrm{M}}$ solution in simulation2

We consider the domain with parameters which are set out in Table 2 and grid spacing for approximated solution Table 3 as following.

Table 3. Convergence of FTCS method ADEM and ADEMM vs grid spacing

\begin{tabular}{|c|c|c|c|c|c|c|}
\hline$\Delta \mathrm{x}$ & $\Delta \mathrm{y}$ & $\Delta \mathrm{t}$ & $\mathrm{T}$ & FTCS & ADEM & ADEM $_{\mathrm{M}}$ \\
\hline 1.0 & 1.0 & 0.1 & 36000 & stable & stable & stable \\
\hline 0.5 & 0.5 & 0.1 & 36000 & stable & stable & stable \\
\hline 0.2 & 0.2 & 0.01 & 36000 & stable & stable & stable \\
\hline 1.0 & 1.0 & 1.0 & 3600 & stable & stable & stable \\
\hline 0.2 & 0.2 & 0.1 & 36000 & unstable & stable & stable \\
\hline 0.2 & 0.2 & 1.0 & 3600 & unstable & stable & stable \\
\hline 0.5 & 0.5 & 1.0 & 3600 & unstable & stable & stable \\
\hline
\end{tabular}

\subsection{Simulation 3: Groundwater in Heterogeneous Aquifer}

Again, we considered a $10 \times 10(\mathrm{~km})$ area - see Fig. 9 . Assumed that the rate of change of hydraulic head along the west boundary is $-0.1(\mathrm{~m})$. Similarly, the rate of change along the north, south and east boundaries are $-0.1(\mathrm{~m}), 0.1$ $(\mathrm{m})$ and $0.0(\mathrm{~m})$ respectively. The potential hydraulic head is $0(\mathrm{~m})$. The storage capacity is $10\left(\mathrm{~m}^{-1}\right)$. We discretize the domain by dividing the interval, $\Delta \mathrm{x}=0.5, \Delta \mathrm{y}=0.5$ and $\Delta \mathrm{t}=$ 0.01 for all $0 \leq x \leq 10,0 \leq y \leq 10$ and $0 \leq z \leq 3600$. We used these parameters in Table 4 and computed solution with heterogeneous hydraulic conductivity function. FTCS, ADEM and $\mathrm{ADEM}_{\mathrm{M}}$ were stable - see Table 5 and total mass error as shown in Table 6.

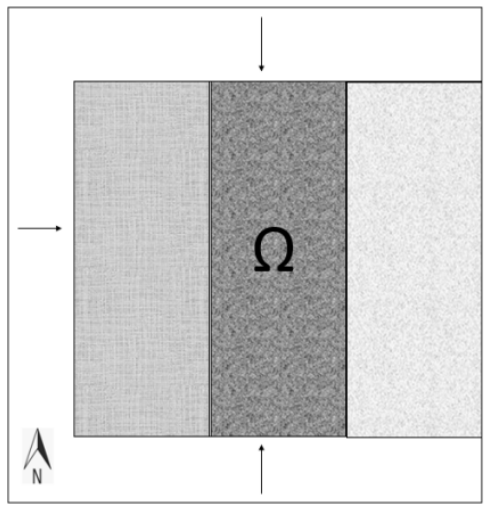

Figure 9. Area for simulation 3 
Table 4. Parameters for simulation 3: Groundwater hydraulic head

\begin{tabular}{|c|c|c|c|c|c|c|c|c|c|c|}
\hline $\mathrm{K}$ & $\mathrm{S}$ & $\mathrm{W}$ & $\mathrm{h}(\mathrm{x}, \mathrm{y}, 0)$ & $\mathrm{B}_{\mathrm{hW}}$ & $\mathrm{B}_{\mathrm{hE}}$ & $\mathrm{B}_{\mathrm{hs}}$ & $\mathrm{B}_{\mathrm{hN}}$ & $\Delta \mathrm{x}$ & $\Delta \mathrm{y}$ & $\Delta \mathrm{t}$ \\
\hline 1.5 & 10 & 0.0 & 0.0 & -0.1 & 0.0 & 01 & -0.1 & 1.0 & 1.0 & 1.0 \\
\hline
\end{tabular}

Table 5. Convergence of FTCS method ADEM and ADEMM in heterogeneous aquifer

\begin{tabular}{|c|c|c|c|}
\hline Heterogeneous function $f(x, y)$ & FTCS & ADEM & ADEM $_{\mathrm{M}}$ \\
\hline$|\boldsymbol{s i n}(\boldsymbol{x y})|+\mathbf{1 . 5}$ & stable & stable & stable \\
\hline $\mathbf{1 . 5}|\sin (\boldsymbol{x y})|$ & stable & stable & stable \\
\hline $\boldsymbol{\operatorname { s i n } ( \boldsymbol { x } y ) ^ { 2 } + \mathbf { 1 . 5 }}$ & stable & stable & stable \\
\hline $\mathbf{1 . 5} \sin (\boldsymbol{x y})^{\mathbf{2}}$ & stable & stable & stable \\
\hline$\sqrt{\boldsymbol{\operatorname { s i n } ( \boldsymbol { x y } ) ^ { 2 }}+\mathbf{1 . 5}}$ & stable & stable & stable \\
\hline $\mathbf{1 . 5} \sqrt{\sin (\boldsymbol{x y})^{2}}$ & stable & stable & stable \\
\hline
\end{tabular}

Table 6. Total mass error for FTCS, ADEM and ADEMM in a heterogeneous aquifer

\begin{tabular}{|c|c|c|c|}
\hline Total mass error & FTCS & ADEM & $\mathrm{ADEM}_{\mathrm{M}}$ \\
\hline$|\sin (x y)|+1.5$ & 6.30 & 6.30 & 6.30 \\
\hline $1.5|\sin (x y)|$ & 9.22 & 8.86 & 9.22 \\
\hline $\sin (x y)^{2}+1.5$ & 4.38 & 1.78 & 4.38 \\
\hline 1. $5 \sin (x y)^{2}$ & 8.64 & 8.22 & 8.64 \\
\hline$\sqrt{\sin (x y)^{2}+1.5}$ & 3.51 & 0.18 & 3.51 \\
\hline $1.5 \sqrt{\sin (x y)^{2}}$ & 9.22 & 8.86 & 9.22 \\
\hline
\end{tabular}

\section{Results and Discussion}

In simulation 1, hydraulic heads for the three methods forward time centered space, traditional and modified alternating direction explicit method were similar - see Fig 3 - Fig 6. In simulation 2, comparing convergence of the solutions, forward time centered space was not able to converge, whereas traditional and modified alternating direction explicit method produced stable solutions - see Table 3. In simulation 3, Groundwater in heterogeneous aquifer, forward time centered space, traditional and modified alternating direction explicit method agreed closely in the heterogeneous aquifer. However, traditional alternating direction explicit method led to more accurate solutions than forward time centered space and modified alternating direction explicit method - as shown in the total mass errors in Table 6.

\section{Conclusions}

We presented a two-dimensional groundwater flow model that simulated the hydraulic head levels well. The equation was used for the groundwater movement.
Simulation of hydraulic head of the groundwater under landfill construction in a rural area showed movement of groundwater, entered to all directions underground in homogeneous and heterogeneous aquifer. All simulations show the groundwater flowed down-gradient from the high hydraulic head to the low. The traditional alternative direction explicit method was modified to form our modified alternative direction explicit method. The results of all method are closed. The traditional alternative direction explicit method and the modified alternative direction explicit method led to more accurate solutions than forward time centered space, two methods ensured that a stable solution of all interior points can be obtained by using a fine grid spacing to simulate a heterogeneous aquifer.

\section{Acknowledgements}

This research is supported by the Centre of Excellence in Mathematics, the Commission on Higher Education, Thailand.

\section{REFERENCES}

[1] Majumdar D. (2003). The Blue Baby Syndrome Nitrate Poisoning in Humans, Resonance, 8(10), 20-30.

[2] Pongnu N, Pochai N. (2011). A numerical computation of non-dimensional from of a mathematical model of soil salinity profile in a rice field near marine shrimp aquaculture farm, Adv. studies Theor.Phy., 5(4), 185-191.

[3] Pongnu N, Pochai N. (2012). A numerical treatment of a couple mathematical model of ground water in rice field near marine shrimp aquaculture farm, Applied mathematical Sciences, 6(6), 283-289.

[4] Schweizer B. (2015). Darcys law and groundwater flow, Snapshots of modern mathematics.

[5] Konig L F, Weiss J L. (2008). Groundwater: Modeling, Management and Contamination, 149-165, New York, Nova Science.

[6] Pongnu N, Pochai N. (2017). Numerical simulation of groundwater measurement using alternating direction methods, Journal of Interdisciplinary Mathematics, 20(2), 513-541.

[7] Karahan H, Ayvaz MT. (2005). Transient groundwater modeling using spreadsheets, Advances in Engineering Software, 36, 374-384.

[8] Anderson MP, Bair ES. (2001). The power of spreadsheet 
models, MODFLOW 2001 and Other Modeling Odysseys Proceedings, International Ground Water Modeling Center,Colorado School of Mines, pp. 815-822.

[9] Anderson MP, Woessner WW. (1992). Applied groundwater modeling: simulation of flow and advective transport, Academic Press, New York.

[10] Pochai N. (2011). A numerical treatment of nondimensional form of water quality model in a nonuniform flow stream using saulyev scheme, Hindawi, Mathematical Problems in engineering.

[11] Shingyu L, Stanley O. (2005) An alternating direction explicit (ADE) scheme for time-dependent evolution equations, Preprint UCLA.

[12] Martin G, Fabrice C, Richard E. (2011) Extensions to Higher-Dimensions of an Unconditionally Stable ADE Scheme for the Convection-Diusion Equation. [Research Report], pp. 21. $<$ hal-00750389v2 $>$. 